Swarthmore College

Works

$1-10-2004$

\title{
Testing Protoplanetary Disk Alignment In Young Binaries
}

Eric L.N. Jensen

Swarthmore College, ejensen1@swarthmore.edu

R. D. Mathieu

A. X. Donar

Allyn Dullighan , '01

Follow this and additional works at: https://works.swarthmore.edu/fac-physics

Part of the Astrophysics and Astronomy Commons

Let us know how access to these works benefits you

\section{Recommended Citation}

Eric L.N. Jensen; R. D. Mathieu; A. X. Donar; and Allyn Dullighan , '01. (2004). "Testing Protoplanetary Disk Alignment In Young Binaries". Astrophysical Journal. Volume 600, Issue 2. 789-803. DOI: 10.1086/380089 https://works.swarthmore.edu/fac-physics/7

This work is brought to you for free by Swarthmore College Libraries' Works. It has been accepted for inclusion in Physics \& Astronomy Faculty Works by an authorized administrator of Works. For more information, please contact myworks@swarthmore.edu. 


\title{
TESTING PROTOPLANETARY DISK ALIGNMENT IN YOUNG BINARIES
}

\author{
ERIC L. N. JENSEN \\ Swarthmore College Department of Physics and Astronomy, Swarthmore, PA 19081; ejensen1@swarthmore.edu \\ Robert D. Mathieu \\ Department of Astronomy, 475 North Charter Street, University of Wisconsin, Madison, WI 53706; mathieu@astro.wisc.edu \\ Arianne X. Donar \\ Wesleyan University; adonar@wesleyan.edu \\ AND \\ Allyn Dullighan \\ Massachusetts Institute of Technology; allyn@space.mit.edu \\ Received 2002 November 7; accepted 2003 September 25
}

\begin{abstract}
We present $K$-band $(2.2 \mu \mathrm{m})$ imaging polarimetry that resolves $19 \mathrm{~T}$ Tauri binary and multiple systems in the Taurus-Auriga and Scorpius-Ophiuchus star-forming regions. We observed systems with projected separations 1."5-7."2 ( 200-1000 AU) in order to determine the relative orientation of the circumstellar disks in each binary system. Scattered light from these disks is polarized, allowing us to deduce the position angle of the disk on the sky from the position angle of polarization even though our observations do not resolve the disks themselves. We detected measurable polarization (typically $0.5 \%-2 \%$, with typical uncertainty $0.1 \%$ ) from both stars in 14 of the systems observed. In eight of the nine binary systems, the two stars' polarization position angles are within $30^{\circ}$ of each other, inconsistent with random orientations. In contrast, the five triple and quadruple systems appear to have random disk orientations when comparing the polarization position angles of the widest pair in the system; the close pairs are unresolved in all but one system. Our observations suggest that disks in wide (200-1000 AU) binaries are aligned with each other within $\leqslant 20^{\circ}$ but not perfectly coplanar. However, we cannot conclusively rule out random relative disk orientations if the observed polarizations are significantly contaminated by interstellar polarization. Even in the presence of interstellar polarization our observations securely exclude coplanar disks. These results provide constraints on possible binary formation mechanisms if the observed orientations are primordial. On the other hand, models of disk-binary interactions indicate that the disks may have had time to decrease their relative inclinations since formation. If the common orientation of the disks in these binaries is a tracer of the binary orbital plane, then our results also have significance for the stability of planetary orbits, suggesting that planetary systems in wide binaries should be stable over $10^{9} \mathrm{yr}$ timescales.
\end{abstract}

Subject headings: circumstellar matter - planetary systems: formation - planetary systems: protoplanetary disks — polarization — stars: formation — stars: pre-main-sequence

\section{INTRODUCTION}

It is now well established that most stars are members of binary systems at birth (see, e.g., recent reviews by Mathieu et al. 2000 and Köhler \& Brandner 2001). Thus, understanding the origin of binaries is vital to understanding the star formation process. The predominance of binaries also means that, based on number of systems alone, most potential sites of planet formation lie in multiple systems. Indeed, many young binaries harbor disks that are quite similar to those found around young single stars (Jensen, Mathieu, \& Fuller 1994, 1996; Osterloh \& Beckwith 1995). However, disks in binary systems clearly present a more complicated environment for planet formation.

While much progress has been made in recent years in determining the disk properties of pre-main-sequence binaries (Mathieu et al. 2000; Dutrey 2001), we still know little about the relative orientations of the disks in a young binary system. The relative disk orientations are of interest for two reasons: they tell us about the origin of the binary (since different binary formation models differ in their predictions of whether or not disks should be aligned; see $\S 5.2$ ), and they tell us about the future stability of any planets that might form in the system (since misaligned systems are less stable on long timescales; Holman \& Wiegert 1999).

Imaging of disks has yielded alignment information in a few pre-main-sequence binaries (e.g., HK Tau; Stapelfeldt et al. 1998; Koresko 1998). To explore disk alignment in a larger sample, we have pursued a program of near-infrared imaging polarimetry of young binaries. The scattered light from a disk is partially polarized, and the position angle of the polarization traces the disk orientation on the sky. Thus, we can determine how the disk around a star is oriented even if we cannot resolve the disk. Below we describe the principle behind the method used, followed by our observations. We then analyze the distribution of relative disk orientations; we find that disks around the two stars in a binary system show a strong tendency toward alignment with each other, while the disks in triples and quadruples do not. We then discuss the implications for binary star formation, for the evolution of disk orientations with time, and for the stability of planets in binary systems.

\section{OBSERVATIONAL METHOD}

The use of polarimetry for studying disk orientations is based on the fact that scattering of starlight in a flattened disk 
or envelope yields a net polarization in the unresolved light. The orientation of the polarization is perpendicular to the disk plane if there is little or no scattering from an extended envelope, or parallel to the disk plane if there is a substantial envelope or bipolar outflow associated with the disk (Whitney \& Hartmann 1992, 1993). In either case, the polarization yields information about the disk orientation even when the disk itself cannot be spatially resolved. In particular, two disks with the same orientation (e.g., two coplanar disks in a binary system) will have the same position angle of polarization in their unresolved light. ${ }^{1}$ Thus, by measuring the polarization of individual components of young binary systems, we can explore whether or not the disks have a common orientation.

This method is complicated by the fact that polarization is insensitive to any tilt of the disk axis into or out of the plane of the sky. The disk is projected as an ellipse on the plane of the sky, and the polarization reveals the position angle of the major axis of that ellipse. Tilting the disk so it is more edge-on or more face-on will increase or decrease the axis ratio of the projected ellipse, but it will not change the orientation of the major axis. Thus, two disks that are not coplanar could in principle have net polarizations whose position angles are the same. (For a diagram illustrating this effect, see Wolf, Stecklum, \& Henning 2001.) Conversely, in the special case in which the disks are viewed close to face-on, even a small orientation difference can result in very different position angles on the sky. However, most of the time a small orientation difference results in a small position angle difference. Exactly coplanar disks will always have the same position angle on the sky.

Because of this projection effect, we cannot conclude securely from this method that any given binary system has aligned disks. However, the influence of this projection effect on a sample of binary systems can be modeled to convert any distribution of true disk orientations in binaries into an observed distribution of polarization position angle differences. We describe such modeling and comparison with our data in $\S 4.2$ below. For additional discussion of this method of determining relative disk orientations in binaries, see Monin, Ménard, \& Duchêne (1998) and Wolf et al. (2001).

\section{OBSERVATIONS AND DATA REDUCTION}

\subsection{Observations}

We observed with the $3.8 \mathrm{~m}$ UKIRT telescope on Mauna Kea on the nights of 1997 December 15 and 1998 April 14-15 using the IRCAM3 camera and IRPOL2 polarimeter with a plate scale of 0.143 per pixel. This instrumental setup provides efficient, high-precision polarimetry because a Wollaston prism is used to image both orthogonal polarizations simultaneously. The normalized Stokes parameters $q$ and $u$ (see $\S 3.2$ ) are then determined from ratios of the two intensities measured in each frame, canceling any changes in sky transmission between images and thus avoiding errors sometimes produced by single-beam polarimeters.

We observed visible young binaries in Taurus-Auriga and Scorpius-Ophiuchus that have projected separations in the

\footnotetext{
${ }^{1}$ In principle, a binary system with coplanar disks could show a polarization position angle difference of $90^{\circ}$ in the unresolved light if the two disks or flattened envelopes differ greatly in their extent perpendicular to the disk plane. In practice, however, binary systems tend to have both components in similar stages of disk evolution (Prato \& Simon 1997; Duchêne et al. 1999), and we do not detect any systems with position angle differences close to $90^{\circ}$.
}

range $1 . .5-10^{\prime \prime} 0$ and that were detected by IRAS at $\lambda=12 \mu \mathrm{m}$. We avoided systems with very high visual extinction by limiting ourselves to systems visible on the Palomar Sky Survey. Basic properties of our sample are given in Table 1. The lower separation bound was chosen so that we could cleanly separate the two components and the upper bound to avoid pairs that are only chance projections (see, e.g., the analysis of Leinert et al. 1993). The IRAS criterion indicates that these systems have circumstellar material; a wealth of direct and indirect evidence (see McCaughrean, Stapelfeldt, \& Close 2000 for a review) indicates that this material lies in flattened circumstellar disks. We observed in the $K$ band $(\lambda=2.2 \mu \mathrm{m})$, where these stars still have ample photospheric emission and where the interstellar polarization is a factor of 3-7 lower than at $V$ (Martin et al. 1992). Each target was observed at four wave-plate angles $\left(0^{\circ}, 22^{\circ} .5,45^{\circ}\right.$, and $\left.67^{\circ} .5\right)$. Each target was observed multiple times (typically 12-18 images at each wave-plate angle), with the target stars jittered to different points on the array to reduce the influence of bad pixels. The typical seeing in the images was 0."5-0"7 (FWHM).

\subsection{Data Reduction}

Data reduction was done primarily using IRAF. ${ }^{2}$ Images were dark-subtracted and flat-fielded using standard techniques. To create flats, we utilized blank parts of the images themselves. Because of the construction of the polarimeter, the target stars occupy only one-half of the CCD in a given image. The other sections (containing only sky background emission) are thus available for constructing flats. By median-filtering hundreds of these unused sections for each of the four waveplate angles we created four flats, one at each wave-plate angle of the polarimeter; each image was then flat-fielded by a flat taken at the same wave-plate angle. Once the data were flat fielded, we removed any occurrences of cosmic rays.

We removed sky background emission by subtracting images from each other that were of the same field and taken near in time but in which the stars were in different positions on the chip. We then performed aperture photometry on the stars in each image, with a circular aperture size chosen for each target to maximize the amount of light encircled while avoiding contamination from the nearby binary companion. The stellar images show no discernible diffraction spikes, so the extent of any possible contamination is a function only of separation and not of position angle. We used aperture radii ranging from 3 to 11 pixels ( $\left.0.4-1{ }^{\prime \prime} 6\right)$, with most of the binary components measured in 5 or 7 pixel $\left(0^{\prime \prime} 7\right.$ or $\left.1^{\prime \prime}\right)$ radius apertures. In systems with separations less than 3.'5, we fitted a radial profile to the brighter of the two stars (using only the half of the star farthest from the secondary) and used the fit to determine the extent of spillover of light from the primary star into apertures of various sizes around the secondary star. We then chose an aperture for which the effect of primary light on the secondary star's polarization measurement would be less than the polarization uncertainty due to image-to-image scatter in the photometry; this latter uncertainty is typically of order $0.05 \%$ in the Stokes parameters $q$ and $u$ in our data (Table 2). In most cases the effect of any possible contamination was substantially less than other error sources. In the few cases where the uncertainty from this possible contamination (under conservative assumptions about the intrinsic polarizations of

\footnotetext{
${ }^{2}$ IRAF is distributed by the National Optical Astronomy Observatory, which is operated by the Association of Universities for Research in Astronomy, Inc., under cooperative agreement with the National Science Foundation.
} 
TABLE 1

Young Binaries OBSERVEd

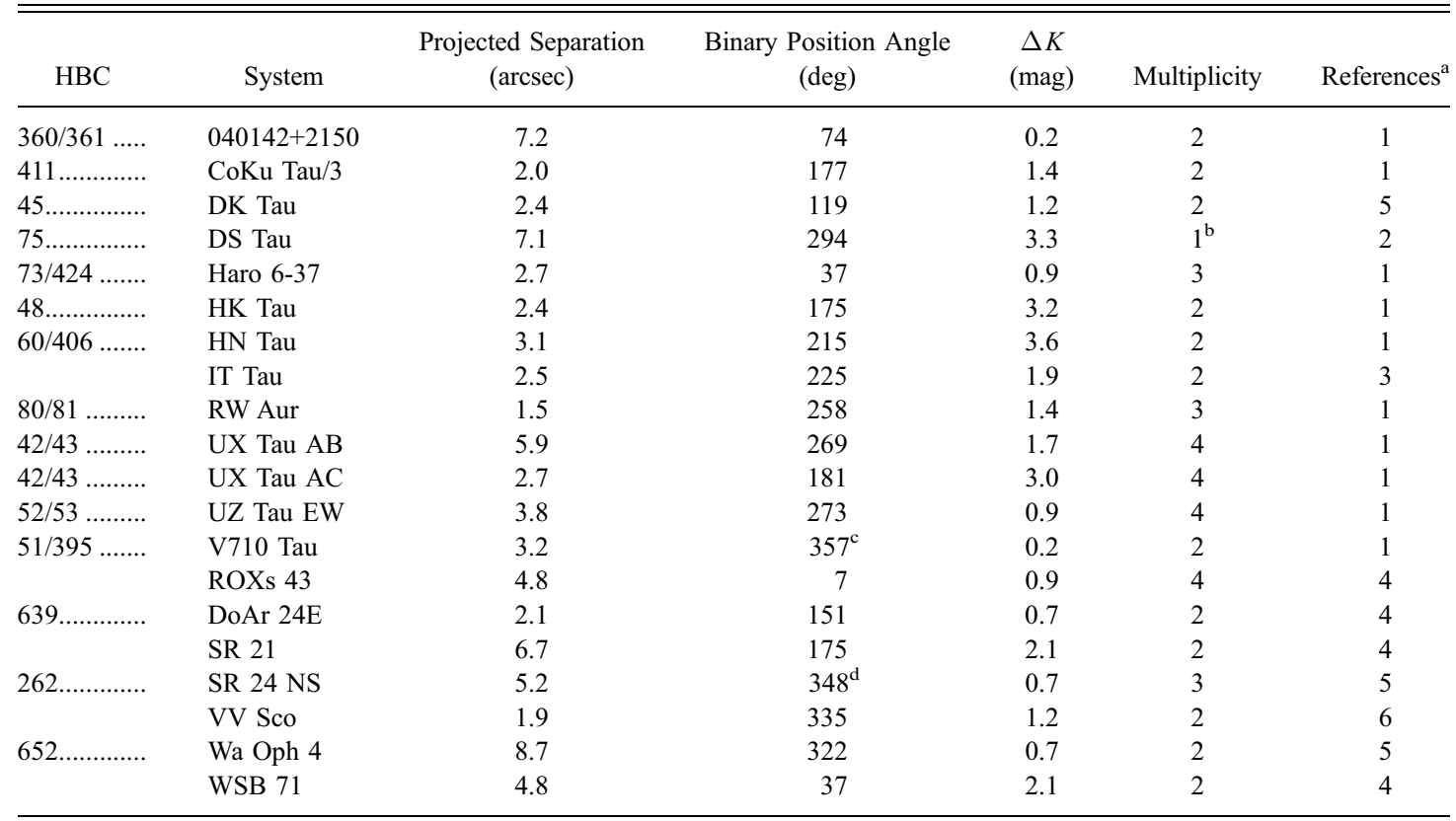

\footnotetext{
a Approximate $K$ magnitude differences are from this work.

b We inadvertently included DS Tau in our target list although Moneti \& Zinnecker 1991 found that the companion to DS Tau is a background field star. We report our polarization observations here, but we do not include DS Tau in the subsequent analysis.

c The southern star is the primary at $K$ band, but the northern star is brighter at visible wavelengths and harbors most of the disk mass in the system (Jensen \& Akeson 2003). In this paper, V710 Tau A refers to the southern star.

${ }^{\mathrm{d}}$ The position angle of $60^{\circ}$ originally given by Herbig \& Bell 1988 and quoted in Ghez, Neugebauer, \& Matthews 1993 and Simon et al. 1995 is in error.

REFERENCES.-For position angle and binary separation: (1) Leinert et al. 1993; (2) Moneti \& Zinnecker 1991; (3) Simon et al. 1992; (4) Simon et al. 1995; (5) Reipurth \& Zinnecker 1993; (6) this work.
}

the two sources) was more than $\frac{1}{10}$ of the uncertainty due to other sources, we added an uncertainty term in the quoted measurements to reflect this. These additional uncertainties are at the few times $0.01 \%$ level (with the largest being $0.06 \%$ ) and have no effect on our conclusions.

For RW Aur, the smallest separation system in our sample at 1.5 , the images of the two stars are not separated sufficiently that we believe we can reliably measure the polarization of each star without contamination of one star by the other. Thus, we report its polarization below but we do not include it in our analysis. The likely effect of contamination would be to make the measured position angle difference of $27^{\circ} .5$ an underestimate of the true position angle difference for this triple system.

\subsection{Polarization Calculations}

The aperture photometry yielded eight flux measurements for each single set of observations of a target star; at each of the four wave-plate angles, we measured the flux in the two orthogonal polarizations, which we will call the $e$ and $o$ (for "extraordinary" and "ordinary") beams. These measurements are combined to produce the normalized Stokes parameters $q$ and $u$ :

$$
q=\frac{Q}{I}=\frac{R_{Q}-1}{R_{Q}+1}, \quad \text { where } R_{Q}^{2}=\frac{(e / o)_{0}}{(e / o)_{45}} .
$$

Similarly,

$$
u=\frac{U}{I}=\frac{R_{U}-1}{R_{U}+1}, \text { where } R_{U}^{2}=\frac{(e / o)_{22.5}}{(e / o)_{67.5}},
$$

where the rightmost subscripts represent the wave-plate angle of the polarimeter for that measurement. Because the $e$ and $o$ fluxes from each exposure are ratioed, this method cancels any difference in sky transmission between exposures.

The observed polarization $p^{\prime}$ and position angle $\theta$ can then be calculated from

$$
p^{\prime}=\sqrt{q^{2}+u^{2}}, \quad \theta=0.5 \tan ^{-1}(u / q) .
$$

The axes of the instrumental system for IRCAM3/IRPOL2 are not aligned exactly north-south. To determine the offset and thus put our polarization position angles on the equatorial system, we measured the observed binary position angles of all of the sources in Table 1 in our instrumental system (except SR 21 and $040152+3016$, systems in which both stars in the binary did not fit on the chip in one observation) and compared them to the binary position angles reported in the literature. Our observed instrumental values are systematically larger than the literature values by a few degrees. A weighted least-squares fit to 14 binaries for which position angles and uncertainties are given in the literature gives a zero-point correction of $-1^{\circ} .9$, which has been applied to our values of polarization position angle $\theta$ reported in Table 3. The Stokes parameters reported in Table 2 are on the instrumental system and have not been corrected. The correction derived here is somewhat smaller than the value of -6.3 suggested by Chrysostomou, ${ }^{3}$ but we note that it gives good agreement not only with the binary position angles measured by others, but also with the polari-

\footnotetext{
${ }^{3}$ See A. Chrysostomou at http://www.jach.hawaii.edu/JACpublic/UKIRT/ instruments/irpol/irpol.html.
} 
TABLE 2

Normalized Stokes Parameters of Young Binaries

\begin{tabular}{|c|c|c|}
\hline System & $\begin{array}{c}q \\
(\%)\end{array}$ & $\begin{array}{c}u \\
(\%)\end{array}$ \\
\hline $040142+2150$ A & $0.130 \pm 0.101$ & $-0.049 \pm 0.081$ \\
\hline $040142+2150$ В .............. & $-0.052 \pm 0.073$ & $-0.093 \pm 0.097$ \\
\hline $\mathrm{CoKu} \mathrm{Tau} / 3 \mathrm{~A} . . . \ldots \ldots \ldots \ldots . . . . . .$. & $-0.251 \pm 0.029$ & $0.880 \pm 0.04$ \\
\hline $\mathrm{CoKu} \mathrm{Tau} / 3$ B.................... & $-0.911 \pm 0.101$ & $1.301 \pm 0.153$ \\
\hline DK Tau A ....................... & $-0.380 \pm 0.016$ & $0.632 \pm 0.02$ \\
\hline DK Tau B ...................... & $0.040 \pm 0.036$ & $-0.921 \pm 0.057$ \\
\hline DS Tau A & $-0.851 \pm 0.030$ & $0.665 \pm 0.024$ \\
\hline 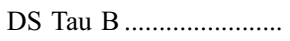 & $-0.712 \pm 0.333$ & $0.255 \pm 0.496$ \\
\hline Haro 6-37 A.... & $0.044 \pm 0.033$ & $0.415 \pm 0.032$ \\
\hline Haro 6-37 В ...................... & $0.116 \pm 0.044$ & $-0.097 \pm 0.043$ \\
\hline HK Tau A & $-0.863 \pm 0.034$ & $-0.570 \pm 0.040$ \\
\hline HK Tau B …................ & $-0.347 \pm 0.317$ & $-4.737 \pm 0.363$ \\
\hline HN Tau A & $-2.021 \pm 0.018$ & $0.117 \pm 0.035$ \\
\hline HN Tau B ....................... & $0.011 \pm 0.485$ & $-0.057 \pm 0.304$ \\
\hline 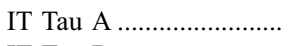 & $0.814 \pm 0.022$ & $0.388 \pm 0.019$ \\
\hline IT Tau B........................... & $1.348 \pm 0.063$ & $0.095 \pm 0.084$ \\
\hline RW Aur $A^{a}$. & $-0.599 \pm 0.066$ & $-0.371 \pm 0.065$ \\
\hline RW Aur $B^{\mathrm{a}} \ldots$ & $-0.059 \pm 0.068$ & $-1.039 \pm 0.060$ \\
\hline UX Tau A & $-0.242 \pm 0.022$ & $0.778 \pm 0.025$ \\
\hline 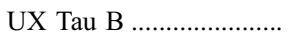 & $-0.030 \pm 0.025$ & $0.100 \pm 0.027$ \\
\hline UX Tau C ....................... & $-0.260 \pm 0.112$ & $0.061 \pm 0.142$ \\
\hline 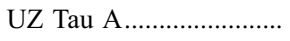 & $0.182 \pm 0.026$ & $0.330 \pm 0.022$ \\
\hline 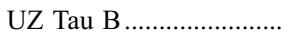 & $1.044 \pm 0.031$ & $0.157 \pm 0.026$ \\
\hline V710 Tau A ...................... & $0.107 \pm 0.050$ & $0.183 \pm 0.030$ \\
\hline V710 Tau B ........................ & $0.435 \pm 0.031$ & $0.271 \pm 0.030$ \\
\hline DoAr 24E A & $0.783 \pm 0.043$ & $0.452 \pm 0.037$ \\
\hline DoAr 24E B..................... & $0.838 \pm 0.157$ & $0.430 \pm 0.191$ \\
\hline ROXs 43 A ...................... & $0.319 \pm 0.046$ & $-0.513 \pm 0.046$ \\
\hline 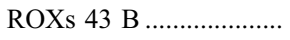 & $0.492 \pm 0.039$ & $0.498 \pm 0.034$ \\
\hline SR 21 A & $0.444 \pm 0.031$ & $0.195 \pm 0.039$ \\
\hline SR 21 B & $0.265 \pm 0.041$ & $0.360 \pm 0.052$ \\
\hline SR 24 A & $-1.011 \pm 0.027$ & $0.886 \pm 0.103$ \\
\hline SR 24 B & $1.028 \pm 0.032$ & $0.482 \pm 0.075$ \\
\hline VV Sco A & $-0.753 \pm 0.026$ & $-0.228 \pm 0.024$ \\
\hline 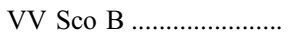 & $-0.050 \pm 0.051$ & $-0.008 \pm 0.071$ \\
\hline Wa Oph 4 A..................... & $0.309 \pm 0.016$ & $0.452 \pm 0.013$ \\
\hline 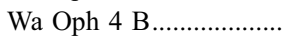 & $0.547 \pm 0.025$ & $0.396 \pm 0.031$ \\
\hline WSB 71 A & $-0.998 \pm 0.029$ & $1.696 \pm 0.024$ \\
\hline 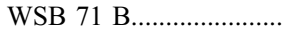 & $0.091 \pm 0.072$ & $1.011 \pm 0.075$ \\
\hline
\end{tabular}

${ }^{\text {a }}$ Tails of stellar images overlap, so measured polarizations may not be independent of each other. This system is not used in subsequent analysis.

zation position angle of the polarized standard star HD 29333, as discussed below. We emphasize that this correction has no effect on the measured position angle differences that form the main part of the analysis below. Thus, we have quoted only the internal uncertainty on the $\theta$ values quoted below, and we have not included any contribution due to the uncertainty of this zero-point correction. This correction adds an additional systematic uncertainty of a few degrees if our individual $\theta$ values (and not just $\theta$ differences) are to be compared to those measured by others.

The observed polarization $p^{\prime}$ is a biased measurement of the true polarization $p$ even when $q$ and $u$ are normally distributed (Simmons \& Stewart 1985). To correct for this bias, we use the prescription of Wardle \& Kronberg (1974) to estimate the polarization. This correction is given approximately by

$$
\hat{p}=p^{\prime}\left[1-\left(\frac{\sigma_{p^{\prime}}}{p^{\prime}}\right)^{2}\right]^{1 / 2},
$$

where $\sigma_{p^{\prime}}$ is the uncertainty on $p^{\prime}$; we use the somewhat more exact polynomial fit given by Stewart (1991). This estimator $\hat{p}$ is a relatively unbiased estimator of the true polarization for signal-to-noise ratios greater than 1 (Simmons \& Stewart 1985). Most of our signal-to-noise ratios are large, so in practice this correction makes little difference for most of our observations.

The precision $\sigma_{p^{\prime}}$ was determined from multiple observations of each star (typically $N=12-18$ exposures at each wave-plate angle), as follows. We calculated $q$ and $u$ values for each of the $N$ observations and then used the mean values $\bar{q}$ and $\bar{u}$ to determine $p^{\prime}$ using equation (3). We calculated $\sigma_{\bar{q}}$ and $\sigma_{\bar{u}}$, the uncertainties on these mean values, as $\sigma_{\bar{q}}=\sigma_{q} / \sqrt{N}$ and $\sigma_{\bar{u}}=\sigma_{u} / \sqrt{N}$, where $\sigma_{q}$ and $\sigma_{u}$ are the standard deviations of the samples of $N$ individual measurements of $q$ and $u$, respectively. These values of $\sigma_{\bar{q}}$ and $\sigma_{\bar{u}}$ for a given star were then added in quadrature with $\sigma_{q_{\text {inst }}}$ and $\sigma_{u_{\text {inst }}}$, the uncertainties on the instrumental polarization (see below), to get the total uncertainty on $q$ and $u$ for that star. We then used 
TABLE 3

Polarization of Young Binaries

\begin{tabular}{|c|c|c|c|c|}
\hline System & $\begin{array}{c}\text { Polarization } \\
(\%)\end{array}$ & $\mathrm{S} / \mathrm{N}$ & $\begin{array}{c}\text { Position Angle } \\
\text { (deg) }\end{array}$ & Number of Observations \\
\hline $040142+2150 \mathrm{~A} \ldots \ldots$ & $0.11 \pm 0.10$ & 1.1 & $167.8 \pm 17.2$ & 8 \\
\hline $040142+2150$ В ...... & $0.07 \pm 0.09$ & 0.8 & $\ldots$ & 8 \\
\hline $\mathrm{CoKu} \mathrm{Tau} / 3 \mathrm{~A} . . . \ldots \ldots$ & $0.91 \pm 0.04$ & 22.6 & $51.1 \pm 0.9$ & 18 \\
\hline $\mathrm{CoKu} \mathrm{Tau} / 3$ B......... & $1.58 \pm 0.14$ & 11.4 & $60.6 \pm 2.2$ & 18 \\
\hline DK Tau A ................... & $0.74 \pm 0.02$ & 37.2 & $58.6 \pm 0.7$ & 18 \\
\hline DK Tau B .................. & $0.92 \pm 0.06$ & 16.2 & $134.3 \pm 1.1$ & 18 \\
\hline DS Tau A ..................... & $1.08 \pm 0.03$ & 38.9 & $69.1 \pm 0.7$ & 18 \\
\hline DS Tau B ................... & $0.68 \pm 0.36$ & 1.9 & $78.2 \pm 18.2$ & 18 \\
\hline Haro 6-37 A............. & $0.42 \pm 0.03$ & 12.9 & $40.1 \pm 2.3$ & 17 \\
\hline Haro 6-37 В ................ & $0.15 \pm 0.04$ & 3.4 & $158.2 \pm 8.2$ & 17 \\
\hline HK Tau A .................. & $1.03 \pm 0.04$ & 28.5 & $104.8 \pm 1.1$ & 12 \\
\hline HK Tau B ................. & $4.74 \pm 0.36$ & 13.1 & $131.0 \pm 1.9$ & 12 \\
\hline HN Tau A ................... & $2.02 \pm 0.02$ & 114.0 & $86.4 \pm 0.5$ & 12 \\
\hline HN Tau B ................. & $0.00 \pm 0.31$ & 0.0 & $\ldots$ & 12 \\
\hline IT Tau A …............... & $0.90 \pm 0.02$ & 41.8 & $10.8 \pm 0.6$ & 18 \\
\hline IT Tau B................... & $1.35 \pm 0.06$ & 21.4 & $0.1 \pm 1.8$ & 18 \\
\hline RW Aur A $\mathrm{A}^{\mathrm{a}} \ldots \ldots \ldots \ldots$ & $0.70 \pm 0.07$ & 10.7 & $104.0 \pm 2.6$ & 11 \\
\hline RW Aur B ${ }^{\mathrm{a}} \ldots \ldots \ldots \ldots . . . . . .$. & $1.04 \pm 0.06$ & 17.2 & $131.5 \pm 1.9$ & 11 \\
\hline UX Tau A .................. & $0.81 \pm 0.02$ & 32.8 & $51.7 \pm 0.8$ & 18 \\
\hline UX Tau B .................. & $0.10 \pm 0.03$ & 3.7 & $51.4 \pm 7.0$ & 18 \\
\hline UX Tau C ................. & $0.24 \pm 0.11$ & 2.1 & $81.5 \pm 15.1$ & 18 \\
\hline UZ Tau A................... & $0.38 \pm 0.02$ & 16.4 & $28.6 \pm 1.9$ & 17 \\
\hline UZ Tau B & $1.06 \pm 0.03$ & 34.0 & $2.4 \pm 0.7$ & 17 \\
\hline V710 Tau A ................ & $0.21 \pm 0.04$ & 5.8 & $28.0 \pm 6.1$ & 18 \\
\hline V710 Tau B ............... & $0.51 \pm 0.03$ & 16.5 & $14.1 \pm 1.7$ & 18 \\
\hline DoAr 24E A ............. & $0.90 \pm 0.04$ & 21.6 & $13.1 \pm 1.2$ & 17 \\
\hline DoAr 24E B.............. & $0.93 \pm 0.16$ & 5.6 & $11.7 \pm 5.6$ & 17 \\
\hline ROXs 43 A ................ & $0.60 \pm 0.05$ & 13.1 & $149.0 \pm 2.2$ & 14 \\
\hline ROXs 43 B .............. & $0.70 \pm 0.04$ & 19.3 & $20.8 \pm 1.5$ & 14 \\
\hline 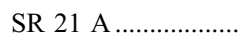 & $0.48 \pm 0.03$ & 15.1 & $10.0 \pm 2.3$ & 18 \\
\hline 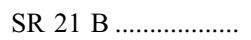 & $0.44 \pm 0.05$ & 9.1 & $24.9 \pm 2.9$ & 17 \\
\hline 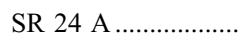 & $1.34 \pm 0.07$ & 18.9 & $67.5 \pm 1.7$ & 13 \\
\hline 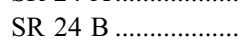 & $1.13 \pm 0.04$ & 26.1 & $10.7 \pm 1.8$ & 13 \\
\hline 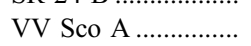 & $0.79 \pm 0.03$ & 30.8 & $96.5 \pm 0.9$ & 26 \\
\hline VV Sco B ................... & $0.00 \pm 0.05$ & 0.0 & $\ldots$ & 26 \\
\hline Wa Oph 4 A................ & $0.55 \pm 0.01$ & 37.9 & $25.9 \pm 0.8$ & 9 \\
\hline Wa Oph 4 B............... & $0.68 \pm 0.03$ & 25.0 & $16.1 \pm 1.2$ & 6 \\
\hline WSB 71 A …............... & $1.97 \pm 0.03$ & 78.1 & $58.3 \pm 0.4$ & 16 \\
\hline WSB 71 B.................. & $1.01 \pm 0.07$ & 13.5 & $40.5 \pm 2.0$ & 16 \\
\hline
\end{tabular}

a Tails of stellar images overlap, so measured polarizations may not be independent of each other. This system is not used in subsequent analysis.

these uncertainties on $q$ and $u$ to calculate the uncertainties $\sigma_{p^{\prime}}$ and $\sigma_{\theta}$ using standard error propagation in equation (3). In the special case where $\sigma_{\bar{q}}=\sigma_{\bar{u}}$, which is nearly true for most of our measurements, $\sigma_{p^{\prime}}=\sigma_{\bar{q}}=\sigma_{\bar{u}}$ and $\sigma_{\theta}=\sigma_{p^{\prime}} / 2 p^{\prime}$ radians. We caution that measurements of $p$ and $\theta$ do not in general follow Gaussian distributions, so that $\sigma_{p^{\prime}}$ and $\sigma_{\theta}$ cannot in general be treated as standard $68.3 \%$ confidence intervals for $\hat{p} / \sigma_{p^{\prime}}<6$ (Simmons \& Stewart 1985; Naghizadeh-Khouei \& Clarke 1993). We discuss confidence intervals more below.

The observed Stokes parameters may contain some component of instrumental polarization introduced by reflections within the telescope and detector optics. We observed the unpolarized standard HD 9540 (Gehrels 1974) during the 1997 run to check the instrumental polarization. We measured $q_{\text {inst }}=-0.094 \% \pm 0.013 \%, u_{\text {inst }}=-0.103 \% \pm 0.014 \%$. This agrees within the uncertainties with the values given by Chrysostomou (see footnote 3 ) of $q_{\text {inst }}=-0.118 \% \pm 0.034 \%$, $u_{\text {inst }}=-0.141 \% \pm 0.032 \%$, and corresponds to a polarization of $0.1 \%$.
In order to measure the extent of any interstellar polarization between Earth and the star-forming regions in which our targets lie, we observed two stars that are projected in front of the Taurus-Auriga cloud, HD 28991 and HD 28819, and one star projected in front of the Sco-Oph region, HD 150937. All three stars are at distances of roughly $100 \pm$ $10 \mathrm{pc}$, as measured by Hipparcos, and thus should lie in front of the clouds, both of which are at distances of approximately 140 pc (Bertout, Robichon, \& Arenou 1999 and references therein). Within the uncertainties, the polarizations measured for the three stars were consistent with each other and with the instrumental polarization determined above. Thus, we conclude that there is substantially less than $0.1 \%$ interstellar polarization in the first $100 \mathrm{pc}$ between Earth and the two observed star-forming regions. We also note that Vrba, Coyne, \& Tapia (1993) found that the interstellar polarization foreground to Ophiuchus was very small.

Because the foreground stars that we observed appear to be completely unpolarized, we combined our observations of 
HD 28819 and HD 28991 with those of HD 9540 to obtain a better estimate of the instrumental polarization, yielding $q_{\text {inst }}=$ $-0.094 \% \pm 0.011 \%, u_{\text {inst }}=-0.093 \% \pm 0.011 \%$, only marginally different from the value obtained from HD 9540 alone. We did not use the observations of HD 150937 because of their somewhat lower signal-to-noise ratio. Observations of the nearby unpolarized standard HD 144287 during the 1998 Ophiuchus run yielded values consistent with these, but with larger uncertainties. Thus, we adopted the instrumental polarization values determined from HD 9540, HD 28991, and HD 28819 for all of our data. These values of $q_{\text {inst }}$ and $u_{\text {inst }}$ were subtracted from the measured $\bar{q}$ and $\bar{u}$ for each target star, and their uncertainties $\sigma_{q_{\text {inst }}}$ and $\sigma_{u_{\text {inst }}}$ were propagated to the final uncertainties on $\hat{p}$ and $\theta$, as described above. We note that there is no evidence for variability of the instrumental polarization with time or with telescope position in our measurements, nor in measurements made by UKIRT staff (A. Chrysostomou 2003, personal communication). There is also no a priori reason to expect any variation of instrumental polarization with telescope position, since UKIRT is an equatorially mounted telescope and thus the optical path through the telescope always maintains the same orientation with respect to the right ascension/declination axes on the sky.

We observed the polarized standards HD 29333 in Taurus and Elias 14 (DoAr 21) in Ophiuchus to check the efficiency of the polarimeter and the position angle calibration. Our measurements of $\hat{p}=0.99 \% \pm 0.02 \%, \theta=74^{\circ} .1 \pm 0.4$ for HD 29333 are in good agreement with those of Whittet et al. (1992) (as modified by Gerakines, Whittet, \& Lazarian 1995 to account for the nonstandard $K$ passband), giving $p=$ $1.03 \% \pm 0.07 \%$ and $\theta=73^{\circ} \pm 3^{\circ}$. The good agreement of our measurements of this standard with previous observations shows that our calibration of the position angle offset is correct, and that the efficiency of the polarimeter is near $100 \%$.

We had hoped to use observations of Elias 14 as an additional check on the calibration, but measurements in the literature suggest that it is polarimetrically variable. Applying the same correction (a Serkowski law) used for HD 29333 to the Whittet et al. (1992) $p$ for Elias 14, using $\lambda_{\max }=0.74 \mu \mathrm{m}$ (Martin et al. 1992) to correct the effective wavelength from 2.04 to $2.2 \mu \mathrm{m}$, gives $p=1.63 \% \pm 0.03 \%$ at $\theta=3^{\circ} \pm 1^{\circ}$, while Martin et al. (1992) measure $p=2.34 \% \pm 0.09 \%$ and $\theta=12^{\circ} \pm 3^{\circ}$. Our measured values are $\hat{p}=1.69 \% \pm 0.02 \%$ at $\theta=7^{\circ} .0 \pm 0.5$ on 1998 April 14 and $\hat{p}=1.72 \% \pm 0.02 \%$ at $\theta=8.0 \pm 0.6$ on 1998 April 15 .

The results of our observations are presented in Tables 2 and 3 . The quoted uncertainties on $q, u, \hat{p}$, and $\theta$ are derived as discussed above. For $q$ and $u$, and for values of $\hat{p}$ and $\theta$ where $\hat{p} / \sigma_{\hat{p}}>6$, these uncertainties can be treated as standard " $1 \sigma$ " $(68.3 \%)$ confidence intervals and can be doubled or tripled to provide the $95.5 \%$ or $99.7 \%$ confidence intervals, respectively. For values of $\hat{p}$ and $\theta$ where $\hat{p} / \sigma_{\hat{p}} \leq 6$, confidence intervals can be derived from the given information using the prescriptions of Stewart (1991) and Naghizadeh-Khouei \& Clarke (1993) for $\hat{p}$ and $\theta$, respectively.

Our primary interest for this work is the position angle difference $\Delta \theta \equiv\left|\theta_{1}-\theta_{2}\right|$ between the polarizations of the two stars in a given binary system, and especially its uncertainty, so that we can gauge whether or not $\Delta \theta>0$. Given normal distributions of $q$ and $u$, the probability distribution of observed $\theta$ values for a given true position angle $\theta_{0}$ is a function of $p, \sigma_{p}$, and $\theta_{0}$, and it is essentially Gaussian for $p / \sigma_{p}>6$ (Naghizadeh-Khouei \& Clarke 1993). However, since in most cases $p / \sigma_{p}$ is not the same for observations of two different stars and in a number of cases it is less than 6 for one component of a binary system, in general it is not true that the uncertainty $\sigma_{\Delta \theta}$ on $\Delta \theta=\left|\theta_{1}-\theta_{2}\right|$ is given by the usual expression $\sigma_{\Delta \theta}^{2}=\sigma_{\theta_{1}}^{2}+\sigma_{\theta_{2}}^{2}$. The analytic expression for the distribution of observed position angle differences is quite complicated and so does not lend itself well to analytic integration to find confidence intervals on $\Delta \theta$. However, it can easily be simulated computationally.

We follow the method of Naghizadeh-Khouei \& Clarke (1993) for calculating the confidence intervals on $\Delta \theta$. For each binary system, we have observed values $\hat{p}_{1}, \hat{p}_{2}, \sigma_{\hat{p}_{1}}, \sigma_{\hat{p}_{2}}, \theta_{1}$, and $\theta_{2}$. For each star in the system, we used the probability distribution of $\theta$ values (eq. [3] of Naghizadeh-Khouei \& Clarke 1993), with the observed $\hat{p}, \sigma_{\hat{p}}$, and $\theta$, to generate more than 350,000 simulated "observed" $\theta$ values. Pairs of points were then randomly selected from the distributions of $\theta_{1}$ and $\theta_{2}$ to generate a sample of the same number of $\Delta \theta$ values for each binary system. This distribution of $\Delta \theta$ values then directly yields confidence intervals on $\Delta \theta$ for that system simply by numerically integrating the distribution to find the range of values of $\Delta \theta$ that encompass the desired percentage of the sample. As expected, in systems where both stars have polarizations with high signal-to-noise ratios (i.e., >6) the confidence levels derived in this way are very similar to those found from simple Gaussian error propagation. In systems where at least one component has a lower signal-to-noise ratio, the $95.5 \%$ and $99.7 \%$ confidence intervals are not integer multiples of the $68.3 \%$ confidence interval, so we have quoted both the $68.3 \%$ and $99.7 \%$ intervals. The sense of the difference is that lower signal-to-noise ratios give $99.7 \%$ confidence intervals that are more than 3 times as large as the $68.3 \%$ intervals. The most extreme case in our data is for UX Tau A and B, where this ratio is 3.6.

Our results are given in Table 4 . The $\Delta \theta$ values quoted reflect the absolute value of the difference in angle of the two polarization vectors, calculated so as to yield a maximum difference of $90^{\circ}$ (since these are two-ended vectors, i.e., a polarization position angle of $0^{\circ}$ is the same as one of $180^{\circ}$ ).

\section{RESULTS AND ANALYSIS}

\subsection{Results}

Our essential results are shown in Figure 1, where we plot $\Delta \theta$ as a function of binary separation for all systems in which both components had detectable polarization with $\hat{p} / \sigma_{\hat{p}} \geq 3$. In the figure we distinguish between systems presently known only to be binary, and triple and quadruple systems. Clearly, a majority of the binary systems cluster at small position angle differences. There is no obvious dependence of $\Delta \theta$ on projected binary separation in the range of 200$1000 \mathrm{AU}$.

In contrast to the binaries, the triple and quadruple systems do not show any tendency toward small position angle differences. The Kolmogorov-Smirnov, Kuiper, and AndersonDarling two-sample tests (see, e.g., Stephens 1974) give probabilities of $14.8 \%, 10.0 \%$, and $12.6 \%$, respectively, that these two subgroups of our data are drawn from the same parent population of position angle differences. While these statistics alone do not overwhelmingly indicate that the two groups must be different, the question that most concerns us here is how each of these groups compares with theoretical expectations. In particular, we would like to test whether or not each of these subgroups of our data is consistent with being drawn from a population of randomly oriented disks. We show below that the 
TABLE 4

Position Angle Differences for Detected Systems

\begin{tabular}{|c|c|c|c|}
\hline System & $\begin{array}{c}\Delta \theta \\
(\operatorname{deg})\end{array}$ & $\begin{array}{c}\sigma_{\Delta \theta} \\
(68.3 \% \text { confidence interval }) \\
(\mathrm{deg})\end{array}$ & $\begin{array}{c}\sigma_{\Delta \theta} \\
(99.7 \% \text { confidence interval }) \\
(\text { deg })\end{array}$ \\
\hline $\mathrm{CoKu} \mathrm{Tau} / 3 \ldots \ldots$ & 9.5 & 2.8 & 8.6 \\
\hline DK Tau ............. & 75.7 & 2.0 & 6.1 \\
\hline Haro $6-37 \ldots \ldots . . .$. & 61.9 & 8.0 & 27.2 \\
\hline HK Tau ............. & 26.2 & 2.5 & 7.4 \\
\hline IT Tau................. & 10.7 & 1.4 & 4.3 \\
\hline UX Tau AB....... & 0.3 & 8.8 & 31.7 \\
\hline UZ Tau.............. & 26.2 & 1.7 & 5.1 \\
\hline V710 Tau ............ & 13.9 & 5.8 & 18.1 \\
\hline DoAr 24E.......... & 1.4 & 5.1 & 15.9 \\
\hline ROXs $43 \ldots \ldots \ldots . . .$. & 51.8 & 2.9 & 8.8 \\
\hline SR $21 \ldots \ldots \ldots \ldots \ldots$ & 14.9 & 3.7 & 11.3 \\
\hline SR $24 \ldots \ldots \ldots \ldots \ldots . . . . .$. & 56.8 & 1.8 & 5.5 \\
\hline Wa Oph 4 .......... & 9.8 & 1.4 & 4.1 \\
\hline WSB $71 \ldots \ldots \ldots \ldots$ & 17.8 & 2.0 & 6.2 \\
\hline
\end{tabular}

triples are quite consistent with being drawn from a random distribution, while the binaries have a very low probability of being drawn from such a distribution.

\subsection{Modeling of the Orientation Difference Distribution}

The results shown in Figure 1 suggest that binary components tend to have disks with similar orientations. However, as noted in $\S 2$, aligned polarization vectors do not unambiguously indicate aligned disks. Thus, more detailed modeling is required to assess the significance of the observed tendency toward aligned polarization vectors among binary systems and the apparently random alignments in the triple and quadruple systems.

In order to compare our data to distributions of disk orientations, we have used Monte Carlo simulations to generate model binary pairs that can then be "observed" to determine

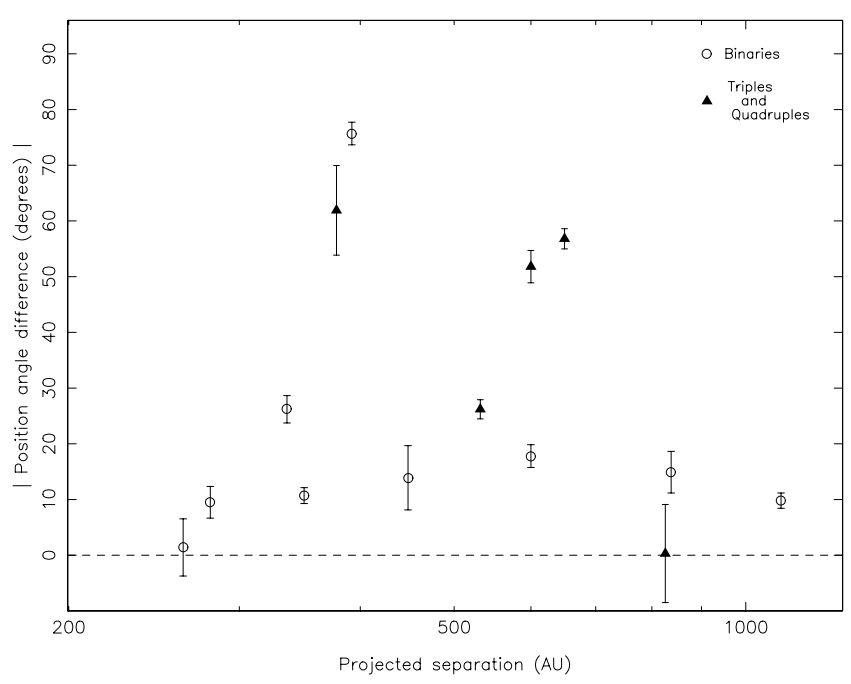

Fig. 1.-Absolute value of the difference in polarization position angles between the two stars in each binary is shown as a function of projected binary separation. Filled triangles show systems that are triple or quadruple. In the majority of the binary systems the two components have similar position angles, indicating that their disks are aligned, while the triple or quadruple systems tend to have more random disk orientations. There is no obvious dependence of alignment on binary separation. the distribution of observed projected angles on the sky resulting from a given distribution of relative disk orientations. In the following discussion we will use $\Delta i$ to denote the true difference of disk orientations for a given binary pair and $\Delta \theta$ to denote the observed difference in the disks' projected position angles on the sky. As noted above, $\Delta \theta$ can be different from $\Delta i$ for a given system; for example, if we take two edge-on disks that are initially coplanar and tilt the axis of one disk $45^{\circ}$ into the plane of the sky (i.e., away from the observer), the observed $\Delta \theta$ will still be $0^{\circ}$ even though $\Delta i$ is now $45^{\circ}$.

A well-known result from studies of galaxy orientations (e.g., Wyatt \& Brown 1955), also shown in Figure 2, is that a random $\Delta i$ distribution (dashed line) produces a uniform $\Delta \theta$ distribution (dotted line), where all $\Delta \theta$ values are equally probable. Thus, the clustering of points at small values of $\Delta \theta$ in Figure 1 is not the result of projection effects; a random distribution of disk orientations should produce a random distribution of points in such a plot.

We generated each model binary system by determining three quantities: the spatial orientation of one disk's axis relative to our line of sight, the azimuthal angle $\phi$ specifying the direction in which the second disk is tilted relative to the first, and the angle $\Delta i$ specifying the amount of tilt. Both the orientation of the first disk and the value of $\phi$ are chosen at random, while $\Delta i$ is chosen from a specified probability distribution $P(\Delta i)$.

The available literature on binary formation provides no specific predictions of the form of the disk orientation distribution $P(\Delta i)$, aside from some models that predict coplanar or parallel disks $(\Delta i=0)$ for all systems and others that predict random disk orientations (see $\S 5.2$ ). Thus we tested these two $\Delta i$ distributions against our data. For random relative disk orientations, we use $P(\Delta i)=\sin (\Delta i)$, accounting for the fact that the amount of solid angle at an angular distance $\Delta i$ from a given point increases as $\sin (\Delta i)$. For correlated disk orientations, we use

$$
P(\Delta i)= \begin{cases}C \sin (\Delta i) & \text { if } \Delta i \leq \Delta i_{\max } \\ 0 & \text { otherwise }\end{cases}
$$

where $C=\left(1-\cos \Delta i_{\max }\right)^{-1}$ is a normalization constant. If $\Delta i_{\max }=0$, this corresponds to all systems being coplanar. 

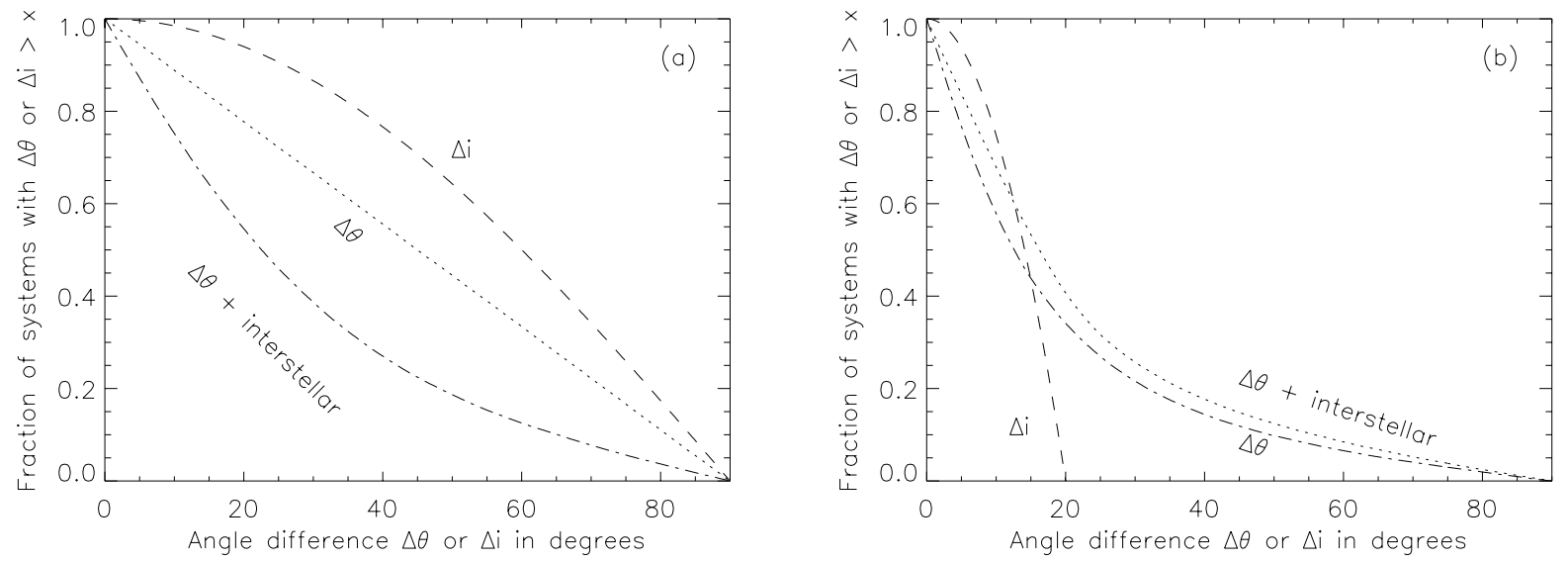

FIG. 2.-Effects of projection in transforming the true distribution of disk orientations $\Delta i$ to the observed distribution of polarization position angle differences $\Delta \theta$. (a) The cumulative distribution function of a random distribution (i.e., proportional to total solid angle at a given $\Delta i$ ) of angle differences $\Delta i$ is shown as a dashed line. This is projected into a uniform distribution of observed position angle differences $\Delta \theta$ (dotted line). Adding an additional component of interstellar polarization (see $\S 4.3$ ) gives a distribution of observed $\Delta \theta$ (dot-dashed line) that is more peaked toward small angle differences. (b) The same comparison, but for an input distribution of angle differences $\Delta i$ (dashed line) that is random from $0^{\circ}-20^{\circ}$. Projection of the disks into the plane of the sky results in some observed position angle differences $\Delta \theta$ that are much larger than any of the true orientation differences $\Delta i($ see $\S 2)$.

However, this distribution (a delta function at $\Delta i=0$ ) clearly does not agree with our data, which show small but mostly nonzero orientation differences. Thus, we explore different values of $\Delta i_{\max }$ below.

For each model binary system, we determined the spatial orientation of the two disks as explained above, then projected these two disks into the plane of the sky to determine the observed $\theta$ value (the position angle of the major axis of the projected disk) for each disk, which is what our polarization observations measure. We created $10^{5}$ model binaries for each $\Delta i$ distribution and then calculated the resulting distribution of $\Delta \theta$ values. These can then be compared directly to our observations. We refer to this as the "hypothesized parent population" below.

To compare the observed $\Delta \theta$ distributions with a hypothesized parent population, we calculated their cumulative distribution functions and then calculated the KolmogorovSmirnov (K-S) statistic $D$, which is the maximum absolute difference between the two cumulative distributions. We determined the probability that our data (consisting of $N_{\text {total }}=$ 14 systems, or the subsets $N_{\text {binary }}=9, N_{\text {triple }}=5$ ) could have been drawn from the hypothesized parent population. Rather than using the standard K-S probability function to assess the significance of a given value of the K-S statistic, we used a computational technique that allows us to include the effect of observational error in drawing samples from the parent population. From the $10^{5}$ model binaries, we generated $10^{5}$ sample "observed" populations of $N$ model binaries each. To simulate the effect of the noise that is present in our actual observations, each $\Delta \theta$ value in the sample population had a random error (which could be positive or negative) $\sigma_{\Delta \theta}$ added to it, with the size of the error determined by assuming a signal-to-noise ratio $p / \sigma_{p}$ and drawing a value from the distribution of $\Delta \theta$ errors calculated as explained in $\S 3.3$ above. The values of $p / \sigma_{p}$ used here are set by those in our observations. Our set of 14 observed systems with detected polarization in both components gives 28 observed values of $p / \sigma_{p}$ (Table 3); these 28 values are used for the 28 stars in each simulated sample of 14 model binaries. Thus each sample of model binaries has the same distribution of "observational" uncertainties as our data.
For each of these "observed" sample populations, we calculated the K-S $D$ statistic to find out how far its cumulative distribution lay from the hypothesized parent population. The null hypothesis is that our data are drawn from the hypothesized parent population. Our confidence level in rejecting this hypothesis (i.e., in concluding that the data are drawn from some other distribution) is then simply given by the fraction of the $10^{5}$ samples that have $D_{\text {sample }} \leq D_{\text {data }}$, where $D_{\text {data }}$ is the K-S statistic calculated by comparing the actual data to the hypothesized parent population and $D_{\text {sample }}$ is the K-S statistic calculated by comparing each sample of $N$ systems of the parent population to the entire parent population. If this fraction is large, then it is rare to randomly choose a sample that is as different from the parent population as our data are, and we have correspondingly high confidence in rejecting that hypothesized parent population. The resulting confidence levels are given in Table 5, and plots of the cumulative distributions are shown in Figure 3. The probabilities were calculated for the entire data set of 14 systems and for the smaller subsamples of only the binaries (nine systems) or only the triple and quadruple systems (five systems). None of these higher order multiple systems is completely resolved; we resolve only the widest pair, and one (or both) of our measured polarization values is for an unresolved pair of stars.

There are a number of other statistics that are similar to the $\mathrm{K}-\mathrm{S}$ statistic but which in some cases have more power in determining whether a data set is drawn from a given distribution (see, e.g., Stephens 1974). For the analysis described above and that which follows, we calculated not only the K-S statistic but also the Kuiper, Watson, Cramer-von Mises, and Anderson-Darling statistics. Unless otherwise noted, these statistics always gave similar results to that from the K-S statistic, so we do not tabulate all of the values of the various statistics.

Considering the data set as a whole (Fig. $3 a$ ), our modeling shows that it is quite unlikely $(0.9 \%$ probability) that our data could have been drawn from an overall population of systems whose disk orientations are randomly distributed in space.

This result is strengthened when we note that most of the systems with larger position angle differences are higher order multiple systems. We divided the data into subsamples of 
TABLE 5

Confidence Level for Rejecting Given Distribution

\begin{tabular}{|c|c|c|c|}
\hline Distribution & $\begin{array}{c}\text { All Data } \\
(\%)\end{array}$ & $\begin{array}{c}\text { Binaries } \\
(\%)\end{array}$ & $\begin{array}{c}\text { Triples/Quadruples } \\
\text { (\%) }\end{array}$ \\
\hline \multicolumn{4}{|c|}{ Observed Polarization Assumed Circumstellar Only } \\
\hline 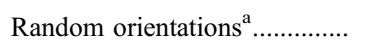 & 99.1 & 99.9 & 38.4 \\
\hline Similar orientations ${ }^{\mathrm{b}} \ldots \ldots \ldots \ldots \ldots$ & 24.4 & 38.0 & 88.6 \\
\hline \multicolumn{4}{|c|}{ Observed Polarization Assumed Interstellar Plus Circumstellar } \\
\hline Random orientations ${ }^{\mathrm{a}} \ldots \ldots \ldots \ldots \ldots . . . . . .$. & 17.8 & 86.4 & 75.3 \\
\hline 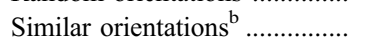 & 74.5 & 64.0 & 93.6 \\
\hline
\end{tabular}

a $P(\Delta i)=\sin (\Delta i)$. This function corresponds to random relative disk inclinations since there is more solid angle at large $\Delta i$.

${ }^{\mathrm{b}} P(\Delta i) \propto \sin (\Delta i)$ for $\Delta i \leq 20^{\circ}, P(\Delta i)=0$ otherwise.
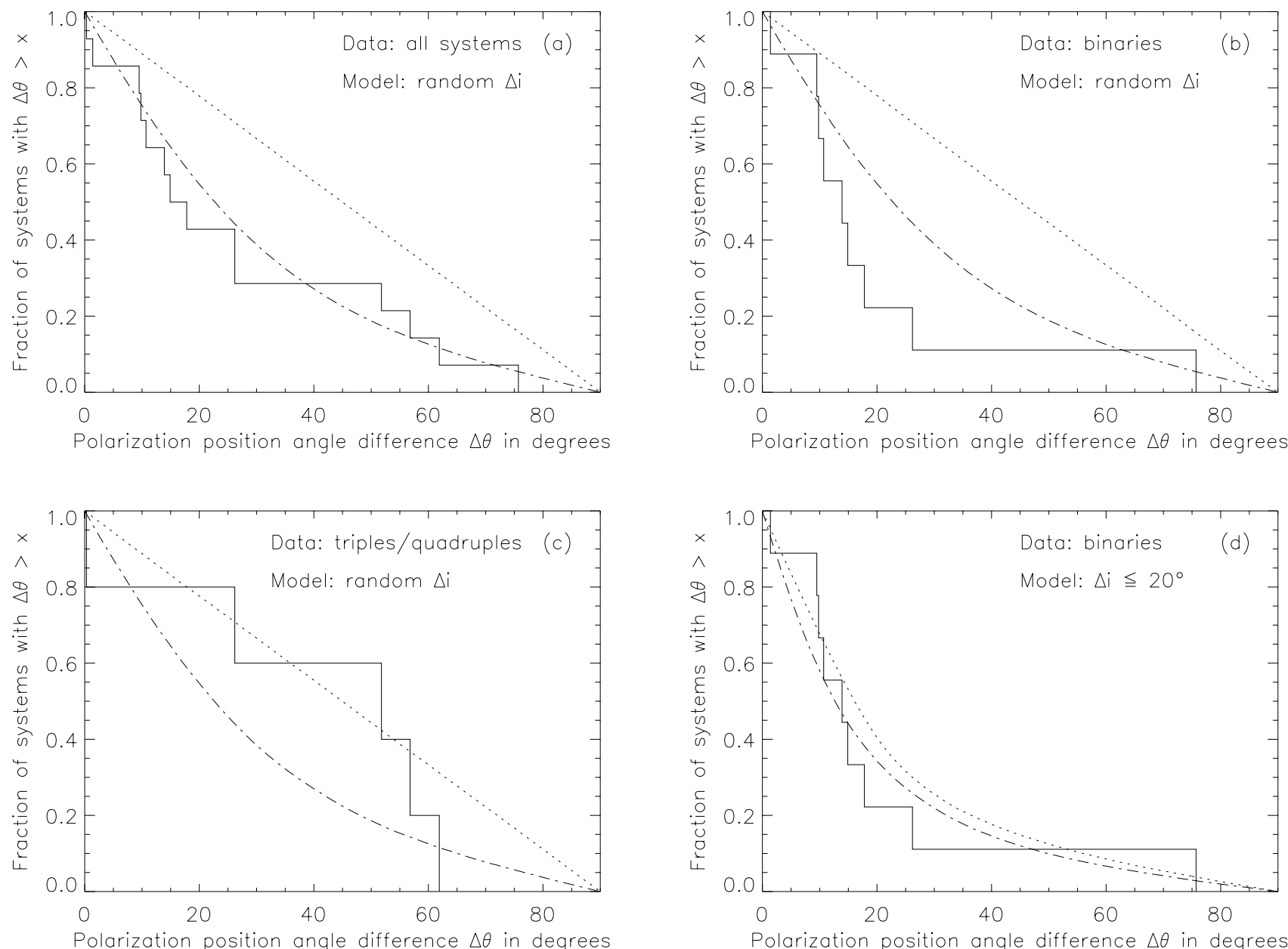

FIg. 3. - Comparison between observed polarization position angle differences and various assumed distributions of angle differences. The cumulative distribution function of the observed polarization position angle differences $\Delta \theta$ (stepped solid line), compared with models with a random distribution of disk orientations (dotted line), and with a random distribution of intrinsic orientations modified by an overlying component of interstellar polarization (dot-dashed line). (a) All data compared with a random distribution. (b) The binaries only, compared to a random distribution. (c) The triples and quadruples (for which only the widest pair is resolved in our observations), compared to a random distribution. $(d)$ The binaries only, compared to a distribution that includes angles only from $\Delta i=0^{\circ}-20^{\circ}$. The binaries are inconsistent with $(b)$ a random distribution but are well fitted by $(d)$ the aligned $0^{\circ}-20^{\circ}$ distribution. In contrast the triples are well fitted by $(c)$ a random distribution. If interstellar polarization is significant (dot-dashed lines), the entire data set is well fitted by $(a)$ a random distribution. See Table 5 for exact confidence levels for the various cases. 
binaries (nine systems) versus triple and quadruple systems (five systems) and repeated the calculations discussed above. The results (Table 5 and Figs. $3 b-3 d$ ) show that the two subsamples differ greatly in how well they match our assumed distributions of $\Delta i$. The binary systems are extremely unlikely $(0.1 \%$ probability) to be drawn from a random distribution of $\Delta i$ values (Fig. $3 b$ ), while the triple systems match such a distribution quite well (62\% probability; Fig. $3 c$ ).

Table 5 and Figure $3 d$ show that the binary systems are well matched by a truncated $\sin \Delta i$ distribution (eq. [5]) with $\Delta i_{\max } \approx 20^{\circ}$. However, neither the functional form of this distribution nor the specific value of $\Delta i_{\max }$ is tightly constrained by our data. We performed the calculations described above with values of $\Delta i_{\max }$ ranging from $1^{\circ}$ to $90^{\circ}$ in $1^{\circ}$ steps, and we find that the best agreement between model and data is obtained for $\Delta i_{\max }$ in the range of $10^{\circ}-30^{\circ}$, with relatively poor agreement (probabilities of $\sim 10 \%$ or less of drawing the binary data from the hypothesized parent population) for larger or smaller values. Other distributions, such as an exponential or a Gaussian, could also agree with our observations if they restrict the range of $\Delta i$. However, distributions strongly peaked at $\Delta i=0^{\circ}$ are not compatible with the data. Thus, while disks show a tendency toward similar orientations, disks are not exactly aligned in most young binary systems.

\subsection{The Influence of Interstellar Polarization}

We have assumed in the analysis above that the observed polarization arises from scattering in the immediate environment of the star, thus tracing the orientation of the circumstellar disk. However, the light we observe must travel through any associated molecular cloud material and also through the diffuse interstellar medium before we measure it. If this intervening material significantly changes the polarization of the light, the polarization signal we measure at Earth would not be representative solely of the orientation of the disk in a given system. Therefore, we have explored the influence that interstellar polarization would have on our data.

\subsubsection{Are We Observing Only Interstellar Polarization?}

As noted in $\S 3.3$ above, observations of stars projected in front of the Taurus-Auriga and Ophiuchus molecular clouds indicate that there is very little interstellar polarization induced by material in the first 100 pc between Earth and the starforming regions. Thus, any interstellar polarization that would contaminate our observations is likely to arise in the clouds themselves.

To explore the influence of the clouds, we compared maps of the cloud polarization (kindly supplied by A. Goodman in electronic form) to the measured position angles of our targets; supplemental polarization data were taken from Vrba, Strom, \& Strom (1976) and Tamura \& Sato (1989). In many cases there is not a measurement of interstellar polarization near our targets. In most cases where the cloud polarization has been measured near our targets, it is not obviously related to the position angle of the polarization we measure. In some cases, there is a large dispersion in position angle among the polarization measurements of stars projected near a given source. In one of our target binaries $(\mathrm{CoKu} \mathrm{Tau} / 3)$, both stars do have polarization position angles that are similar to the well-defined local cloud polarization; however, the two stars in this system show very different levels of polarization (Table 3). Two other systems, DoAr 24E and SR 21, have polarization position angles similar to each other and similar to at least some of the nearby cloud polarization angles, although the cloud angles have a fairly large dispersion. Thus, the source of the apparent alignment in these binaries is open to question, although we note that in a large enough sample there will be some chance alignments of circumstellar and interstellar polarization vectors as well.

More generally, what influence would interstellar polarization be expected to have on our observations? If the intrinsic circumstellar polarization from our targets were zero, then any polarization observed would be only interstellar in origin. This is unlikely for several reasons:

1. All of our targets have infrared excesses.-We have chosen targets that were detected by IRAS and which thus clearly have circumstellar material. Circumstellar material around young stars lies in flattened disks (McCaughrean et al. 2000 and references therein). Scattering from this material is likely to induce some polarization, as infrared excess is correlated with polarization for cool stars in general (Dyck et al. 1971) and for young stars in particular (e.g., Bastien 1985; Yudin 2000). Bastien (1985) also found no correlation between polarization and a star being associated with nebulosity, indicating that the measured polarization of T Tauri stars is circumstellar in origin.

2. Few of our targets have $\Delta \theta=0$.- Interstellar polarization in these clouds is observed to be relatively smoothly varying in strength and position angle on parsec size scales (Vrba et al. 1976; Tamura \& Sato 1989; Goodman et al. 1990); this suggests that the interstellar polarization should change little on the 2001100 AU size scales of the binary separations of our targets, giving $\Delta \theta$ values that are close to zero. However, only two of our targets (DoAr 24E and UX Tau AB) have $\Delta \theta$ within $1 \sigma$ of zero. The larger $\Delta \theta$ values in the other systems indicates at least some variation in the polarizing material on much smaller scales, most likely from differences in the circumstellar disks around the two stars in a binary. Notably, one of the $\Delta \theta \approx 0^{\circ}$ systems (DoAr $24 \mathrm{E}$ ) is one of the binaries already suspected of being influenced by interstellar polarization because of the similarity between its polarization position angle and that of the nearby cloud.

3. Primary and secondary stars have different percent polarizations. - If the observed polarization is mostly interstellar in origin and thus correlated on large length scales, it is surprising that there is little correlation between the percent polarization detected in the two stars in each system. As noted above, maps of interstellar polarization show similar percent polarizations on parsec length scales (about 0.4 for these clouds). Similarly, in the wide binaries observed by Monin et al. (1998), the systems that they suspect of being contaminated by interstellar polarization tend to have similar percent polarizations for the two stars in each pair. We observe no such tendency for pairs with small $\Delta \theta$ to have $p_{\min } / p_{\max }$ close to 1 . However, the two pairs noted above, DoAr 24E and SR 21, do have $p_{\min } / p_{\max }=$ $0.97 \pm 0.17$ and $0.92 \pm 0.12$, respectively, the two largest values in the sample and the only two that have ratios consistent with unity within the uncertainties.

4. The measured polarization is uncorrelated with extinction.-We also find no correlation between visual extinction and percent polarization for the stars in our sample. We separately considered resolved primary/secondary star extinction values from White \& Ghez (2001) and unresolved values from a variety of sources; in neither case did we find a correlation. Extinction is correlated with percent polarization for intrinsically unpolarized stars extincted by the diffuse interstellar medium (e.g., Jones 1989); a correlation is also seen 
in the Taurus clouds up to extinctions of $A_{V}=1.3 \pm 0.2 \mathrm{mag}$ (Arce et al. 1998). We see no correlation in our data, whether for low or high $A_{V}$.

5. Binaries and triples show different behavior.-Finally, the apparent difference between the $\Delta \theta$ distributions for binaries and triples at the $85 \%-90 \%$ confidence level $(\S 4.1)$ suggests that the observed polarization is not primarily interstellar in origin, since there would be no reason for the interstellar material to be different in front of systems of different multiplicity.

Thus, several different lines of reasoning suggest that the polarization we measure is not solely a result of the intervening interstellar medium; at least some of it must be circumstellar in origin.

\subsubsection{Are We Observing a Combination of Interstellar and Circumstellar Polarization?}

However, even if an interstellar contribution to the polarization does not dominate the net polarization, it could still affect our results if the interstellar and circumstellar contributions are similar in magnitude.

If the observed polarization is a combination of both interstellar and circumstellar polarization, the situation is more complicated, since the interstellar polarization can either increase or decrease the value of $\Delta \theta$. To see this, consider a binary system in which the two disks are aligned, so that $\Delta \theta_{\text {circumstellar }}=0$, but in which the two stars have different levels of polarization $\left(p_{1} \neq p_{2}\right)$. Since polarizations add as vectors, adding the same interstellar polarization vector to both components will increase the value of $\Delta \theta$, since it will change the position angle of a small intrinsic polarization vector more than a large one. On the other hand, if the two stars have the same level of polarization, they will be influenced equally by a given interstellar polarization.

We do not have an independent measure of the interstellar polarization for each star. Indeed, we cannot obtain such a quantity, since it depends on the stars' depth within the cloud and we do not have an unpolarized probe of only the relevant part of the sight line. Polarization measurements of stars projected near the same line of sight, but which are located behind or in front of the cloud, will not yield the desired information because they sample too much or too little of the material along the line of sight. Thus, it is impossible to disentangle the influence of circumstellar and interstellar polarization for each of our targets. However, we can explore the influence of plausible values of interstellar polarization on the overall alignment distribution of our data by using the same type of modeling described above.

We wish to test the hypothesis that the distribution of $\Delta i$ values for the binaries is in fact random, but that the influence of interstellar polarization leads to observed $\Delta \theta$ values that are similar to those we observe. To model the influence of interstellar polarization, we added an additional step in the construction of model binary systems described above. For each model binary, we chose a Stokes vector $\left(q_{\text {is }}, u_{\text {is }}\right)$ representing interstellar polarization, as well as a percent polarization for the circumstellar polarization; the position angle of the circumstellar polarization was set by the orientation of the disk. The interstellar polarization vector was taken to be the same for both stars in a system, while the circumstellar polarization was not assumed to be the same either in magnitude or direction for the two stars. We added the circumstellar and interstellar polarization Stokes vectors for each component in the system to get its total "observed" polarization and position angle. We then used the difference in orientation of these total polarization vectors to determine the observed $\Delta \theta$ for each system as described above.

The values we choose for the interstellar and circumstellar polarization components have some constraints. First, the distribution of total (circumstellar plus interstellar) polarization values in the modeling must be similar to that seen in the data, as must the ratios $p_{\min } / p_{\max }$. Second, there is little point in choosing a very small interstellar polarization value compared to the circumstellar polarization, since this would have little effect on the modeling discussed above. Finally, we cannot set the circumstellar polarization to zero, since (under our hypothesis that interstellar polarization does not vary on $\sim 1000$ AU scales) this would lead to $\Delta \theta$ values too small to match the data.

Taking these constraints into account, we tested various combinations of circumstellar and interstellar contributions to the total polarization. We chose the contributions to yield a range of net polarizations from $0 \%$ to $2.2 \%$, similar to the range of observed polarizations in our sample with the exception of HK Tau B, which is known to have anomalously high extinction due to an edge-on circumstellar disk (Stapelfeldt et al. 1998; Koresko 1998). To parameterize this simply, we assumed that the circumstellar polarization contribution was uniformly distributed in the interval $0 \%$ to $f_{\mathrm{cs}} \times 2.2 \%$, and that the interstellar polarization contribution was uniformly distributed in the interval $0 \%$ to $\left(1-f_{\mathrm{cs}}\right) \times 2.2 \%$. Here $f_{\mathrm{cs}}$ represents the average fraction of the total observed polarization that is circumstellar in origin; $f_{\text {cs }}=1$ corresponds to only circumstellar polarization with no interstellar contribution, while $f_{\mathrm{cs}}=0$ corresponds to only interstellar polarization. We find that values of $f_{\text {cs }} \approx 0.4-0.6$ match the data well, in the sense of making a random distribution of relative disk inclinations match the observed trend of small polarization position angle differences.

The results of this modeling can be seen in the dot-dashed lines in Figure 3 and in Table 5, which show the results for $f_{\text {cs }}=0.5$. For the entire sample (binaries and triples together), the addition of interstellar polarization at an average level of half the total observed polarization raises the acceptability of the random distribution significantly so that it is a very good match to the observed data (Fig. $3 a$ ).

The question, then, is whether this range of interstellar and circumstellar polarization values is plausible given other properties of our data set. We compared the empirical distribution functions of several quantities in our data set to those found in the simulations, including the ratio $p_{\min } / p_{\max }$ for each system, the values of $p_{1}$ and $p_{2}$ individually, and a linear combination of $p_{\min } / p_{\max }$ and $\Delta \theta / 90^{\circ}$. The latter quantity allows us to check for correlation between these quantities using nonparametric two-sample tests. In each case, the quantities in the simulations are consistent with being drawn from the same distribution as those in the data (K-S probabilities of $20 \%$ or more). Finally, this range of interstellar polarization values is similar to that observed by Vrba et al. (1993) for Ophiuchus and slightly higher than that observed by Arce et al. (1998) for Taurus. In both cases we made this comparison by using a Serkowski law (e.g., Whittet et al. 2001) to extrapolate the optical polarization measurements to $\lambda=2.2 \mu \mathrm{m}$. Thus, it appears that the model incorporating some interstellar polarization contribution cannot be ruled out with the given data set.

The above analysis assumes that the interstellar polarization and the circumstellar polarization are randomly oriented with respect to each other. This is equivalent to assuming that the 
disk orientations (traced by circumstellar polarization) are independent of the cloud magnetic field orientation (traced by interstellar polarization). However, this may not be the case. Theoretically, magnetic fields are thought to play an important role in the collapse of cloud cores (e.g., McKee et al. 1993; Galli \& Shu 1993; Desch \& Mouschovias 2001 and references therein). Observationally, cloud cores are found to be elongated along the filaments of the Taurus cloud (Myers et al. 1991; Lee \& Myers 1999) and the Ophiuchus cloud (Tachihara, Mizuno, \& Fukui 2000). At later stages of star formation, a number of workers have suggested that the orientation of disks, jets, and/or outflows in star-forming regions is related to the local magnetic field, but not all studies show this, and the observational data are complicated; see Heiles et al. (1993) for a review. Most recently, Duchêne \& Ménard (2004) suggest that the orientations of disks (as determined from direct images of disks and/or jets) around young stars in Taurus are related to the local magnetic field direction as traced by polarization of background stars. Puzzlingly, however, they find that stars with jets have symmetry axes preferentially aligned parallel to the local magnetic field, while those with only disks have symmetry axes preferentially aligned perpendicular to the field. When these two groups are considered together, their orientations are consistent with a random distribution. If disk orientation is indeed related to the local magnetic field direction and thus to the orientation of interstellar polarization, then the problem of contamination by interstellar polarization is not nearly as severe as suggested above.

Our data are thus consistent with two different interpretations. If the observed polarization does not have significant interstellar contamination, the data indicate that binaries tend to have roughly aligned disks, while triples and quadruples tend to have randomly oriented disks.

Alternatively, if there is significant contamination by interstellar polarization (at an average level roughly equal to the circumstellar polarization), and if this contaminating polarization is unrelated to the disk orientations, the data are consistent with randomly oriented disks for all systems. In this scenario, the apparent difference (at the $85 \%-90 \%$ confidence level) between the binaries and triples is simply an artifact of the small sample size, not a real difference.

We note, however, that in either scenario, the data are inconsistent with the disks in binary and multiple systems being exactly coplanar. Models with $\Delta i_{\max } \leq 10^{\circ}$ have probabilities of less than $1.5 \%$ of reproducing the observations if interstellar contamination is unimportant, or less than $5 \%$ if there is contaminating interstellar polarization at the level modeled here. In either case the disks in these systems must be misaligned in general with a distribution of $\Delta \theta$ that extends up to at least $10^{\circ}$.

We find that we cannot conclusively rule out contamination by interstellar polarization as the source of the observed alignment. Our simulations suggest that this is a problem not just in our data but with this technique in general, since the interstellar polarization contribution is not well known on a system-by-system basis. To conclusively subtract the interstellar contribution to a given system's polarization, it is necessary to know how deeply embedded in the cloud it is. We note that our ability to rule out contamination would be stronger with a larger sample size, but only if the difference between the alignment distribution of binaries and triple/quadruple systems $(85 \%-90 \%$ significant in our data) is real; if both types of systems have the same alignment distribution, then the problem with interstellar polarization will persist. As we have shown, it is much easier to prove misalignment than alignment using this technique.

\section{DISCUSSION}

We have shown above that the two circumstellar disks in a young binary system are not perfectly aligned with each other. In addition, if interstellar polarization is not a significant contaminant, the disks in a binary are not randomly oriented but rather appear to have orientations that are typically within $20^{\circ}$ of each other, while in triple and quadruple systems the disks appear to be randomly oriented. Here we compare this result with previous work, and we then discuss its implications. In this discussion, we first assume that the observed distribution of disk orientations is primordial (i.e., that it reflects the state in which the binary pairs formed) and discuss what our observations imply about binary star formation. We then consider whether the disks' orientations could have evolved with time.

\subsection{Comparison with Previous Work}

\subsubsection{Disk Orientations in Young Binary Systems}

The work that is most directly comparable to the results presented here is that of Monin et al. (1998), Wolf et al. (2001), and Monin et al. (2002), who have pursued observational programs independent of our own using the same technique. While these groups have also observed T Tauri stars, as we have, for the most part our samples are independent of each other, since Monin et al. (1998) detected polarization only from the widest pairs in Taurus $\left(88^{\prime \prime} 7-37^{\prime \prime} .3\right)$ and Wolf et al. (2001) observed only in southern star-forming regions.

Monin et al. (1998) measured three pairs in which they were confident that the observed polarization was not contaminated by interstellar polarization. These systems had $\Delta \theta=2^{\circ} \pm 4^{\circ}$ for FZ Tau and FY Tau, $\Delta \theta=28^{\circ} \pm 16^{\circ}$ for V773 Tau and FM Tau, and $\Delta \theta=75^{\circ} \pm 6^{\circ}$ for GI Tau and GK Tau. Thus, one system is relatively closely aligned, one system is clearly misaligned, and one system (given the large uncertainties) could be either. However, we note that two of these three are not simple binary systems but are higher order multiples. V773 Tau is itself a triple system (Welty 1995); its large error bar is consistent with alignment, as noted by Monin et al. (1998), but also with misalignment of $40^{\circ}$ or more. GK Tau is a binary with a separation of 2 ."5 (Reipurth \& Zinnecker 1993), and notably the GK/GI Tau triple system is the most misaligned of the three systems. Thus, the results of Monin et al. (1998) are consistent with what we have found in our larger sample.

Wolf et al. (2001) observed 49 binary or multiple systems with separations $0.5-5$.'3. They detected polarization in both components of 34 of these systems, of which 21 systems have $\Delta \theta \leq 36^{\circ}$, inconsistent with randomly oriented disks. They assert that interstellar polarization is not a major contaminant in their data. They do not analyze the binaries and triples separately. Again, our results are consistent with theirs.

Monin, Ménard, \& Peretto (2002) present preliminary results for 20 systems. They do not present results for individual systems, but they state that their results show that "disks tend to be aligned" in young binaries.

\subsubsection{Orbital Plane Orientation in Main-Sequence Triple and Quadruple Systems}

In systems with three or more stars, one can compare the orientations of the different orbital planes in the system. Several authors have considered whether the short-period 
and long-period orbits in main-sequence triple systems are coplanar. Van Albada (1968) found little tendency toward coplanarity in nine systems, most of which had long periods greater than $100 \mathrm{yr}$ (i.e., periods similar to those in our sample). Fekel (1981) considered only systems with periods less than $100 \mathrm{yr}$ and found that seven of 21 mostly early-type systems were not coplanar $\left(\Delta i \geq 15^{\circ}\right)$, with coplanarity permitted (but not required) for the remaining systems.

The most extensive study is that of Tokovinin (1993; see also Sterzik \& Tokovinin 2002), who found that the data for 45 multiple systems are consistent neither with coplanarity nor with random relative orientations. The misalignments tend to be greater than $20^{\circ}$ but are not random. The data are consistent with a model in which half of the systems are aligned within $20^{\circ}-40^{\circ}$ and the other half are randomly oriented.

\subsection{Binary Formation}

Our observations suggest a tendency for binary systems' disks to be nearly (but not exactly) aligned with each other, and for those in triple and quadruple systems to be misaligned. If these alignments have not been disturbed by dynamical evolution and thus are signatures of the formation processes, it is of interest to ask what constraints these observations may place on binary formation mechanisms. We note that our results probably do not reflect on disk fragmentation models given that the binary separations in our sample are all greater than $200 \mathrm{AU}$ and so are larger than a typical disk size.

Bate et al. (2000) have recently reviewed the implications of disk alignment on binary formation mechanisms, to which the reader is recommended. Simplistically, binary formation scenarios based on cloud fragmentation have been suggested to produce aligned circumstellar disks, while capture scenarios produce arbitrary disk alignments. Interestingly, the thrust of the Bate et al. discussion, building on rich theoretical explorations of the past decade (e.g., Bonnell \& Bastien 1993; Hall, Clarke, \& Pringle 1996), is that while most fragmentation calculations have produced binaries with aligned circumstellar disks, it is "trivial to produce initial conditions" that result in misaligned disks (Bate et al. 2000). Furthermore, there are numerous processes during the protostellar accretion phase (e.g., accretion, stellar encounters, precession) that could produce misaligned circumstellar disks in wide binaries (separations $>100$ AU). Thus the present theory of binary formation is not highly predictive with respect to disk orientation.

Nonetheless, given that there are many formation routes to misaligned disks in wide binaries, our finding that the disks of young binaries typically are very nearly aligned, if correct, suggests either that these processes in fact do not operate or that there is evolution subsequent to the protostellar accretion stage that acts to realign these disks. Given present estimates of evolutionary timescales, we suspect the latter is the case, as we discuss in the next section.

It is also of interest to consider whether the formation of higher order multiple systems is related to the seeming lack of alignment of disks around the widest pairs in these systems. We presume that the circumstellar disks associated with the close binaries in multiple systems are aligned with the binary orbital planes of the close pairs given the short timescales for dynamical alignment (Bate et al. 2000) and that the observed polarized emission results from scattering off of these circumstellar disks rather than circumbinary disks. Our results thus suggest that the orbital planes of the close binaries in multiple systems are not aligned with the orbital planes of the wide pairings. This indicates that the lack of alignment seen among main-sequence multiple systems ( $(5.1 .2)$ is established at a very young age. We suggest that this result may also be evidence that the natal clouds from which multiple systems form have large coupled spatial fluctuations in both their mass and angular momentum distributions, leading to multiple fragmentations with misaligned orbital planes.

\subsection{Evolution of Disk Orientations}

If a disk in a binary system is not coplanar with the binary orbital plane, tidal torques from the companion star will cause the disk orientation to change (Papaloizou \& Terquem 1995; Larwood et al. 1996; Bate et al. 2000; Lubow \& Ogilvie 2000). Thus, we must consider the possibility that the distribution of disk orientations that we observe has changed since the binaries formed.

We first note that we observe no correlation of the relative disk orientation $\Delta \theta$ with binary separation. Since the timescale on which the disk orientation evolves is predicted to be related to the binary orbital period, we note that our observed range of separations corresponds to about an order of magnitude range of orbital periods. The lack of correlation of disk alignment with binary separation implies that the evolutionary timescale must either be much shorter than the $\sim 10^{6} \mathrm{yr}$ age of these binary systems (so that the evolution has already occurred even for the widest separations) or that it is much longer (so that little evolution has yet had time to occur, even for the closest systems). With the caveat that specific predictions of timescales are uncertain because of the poorly known values of disk parameters like viscosity, below we attempt to compare predicted timescales from the literature with our observations.

If disks in a binary system are formed with substantial inclination to the binary orbital plane, nonlinear effects may cause the inclination to decay to a relatively small value in a time that is of order the precessional timescale of the disk, or roughly 20 binary orbits (Bate et al. 2000; Lubow \& Ogilvie 2000). The binaries in our sample have separations of roughly 280-1100 AU; assuming two one-solar-mass stars in each system, this corresponds to orbital periods of $3.3 \times 10^{3}$ to $2.5 \times 10^{4} \mathrm{yr}$. Since all of our targets have ages of order $10^{6} \mathrm{yr}$, even the widest systems we observed have orbited more than 20 times, implying that any large tilts have had time to decay if the timescales given here are correct.

Smaller disk tilts are predicted to decay on the much longer viscous timescale of the disk, of order $10^{3}$ binary orbital periods for a reasonable choice of the disk viscosity (Lubow \& Ogilvie 2000). For the binaries observed here, this corresponds to $3.3 \times 10^{6} \mathrm{yr}$ for the closest binaries, up to $2.5 \times 10^{7} \mathrm{yr}$ for the widest. The short end of this timescale is close to the age of the observed systems, implying that decay to complete coplanarity would not yet have occurred for most of our sample if there was some initial inclination of one or both disks.

The theory allows for the growth of disk tilt with time, but only if the disks in these binaries have radii smaller than 0.1 times the binary separation (Lubow \& Ogilvie 2000). However, such small disks seem unlikely based on millimeter interferometric images that show disk radii of $\sim 200 \mathrm{AU}$ around young single stars (Koerner \& Sargent 1995; Dutrey et al. 1996) and in the wide binary UZ Tauri (Jensen, Koerner, \& Mathieu 1996), and theoretical predictions that disk truncation in closer binaries should occur at $0.3-0.5$ times the binary separation (e.g., Artymowicz \& Lubow 1994). 
If correct, the two tilt decay timescales presented above suggest a possible explanation for a puzzling feature of our data, namely, that the disks may show a tendency toward alignment, and yet few of them are completely aligned. The $10^{4}-10^{5} \mathrm{yr}$ timescale for rapid decay of large tilts and the $10^{6}$ $10^{7} \mathrm{yr}$ timescale for the decay of a small remaining tilt neatly bracket the $10^{6} \mathrm{yr}$ ages of our targets, and so we could be seeing these binaries between the two phases of their disk inclination evolution. Of course, the fact that large initial tilts have had time to decay does not mean that such tilts were initially present, merely that they could have been. Whichever hypothesis is correct, some initial tilt appears to be required by our data to explain the observed predominance of $\sim 10^{\circ}-20^{\circ}$ misalignments.

We note that attributing the present distribution of relative disk alignments to dynamical evolution may also explain the differences in disk alignments that we may have found between binaries and higher order multiple systems. In a binary system the rapid alignment of disks around each star is the result of a coupling between the stellar orbital motions and the internal disk orbits. However, in a higher order system, the binary orbits themselves must be aligned in order for all of the circumstellar disks also to be aligned. If when multiple systems form the internal orbital planes of the close binaries are not aligned with either the orbital planes of the tertiary stars (in triple systems) or the orbital planes of the close binaries about each other (in quadruple systems), then the relevant timescale for disk alignment is not the disk alignment timescale itself but the binary orbit alignment timescale. The latter is much longer than the age of the young binaries that we have observed, and indeed it is apparently longer than main-sequence lifetimes (see $\S$ 5.1.2).

\section{CONCLUSIONS}

We have used infrared imaging polarimetry to probe the alignment of circumstellar disks in young binary systems. If the polarization we measure arises mostly from scattering off circumstellar disks, we find that the disks in young binaries with separations of $280-1100$ AU have relative inclinations distributed between $0^{\circ}$ and $20^{\circ}$. In contrast, the disks of the widest pairings in triple and quadruple systems tend to be randomly oriented.

Alternatively, based on our modeling, there is a chance that our observations could be drawn from binaries with a random distribution of disk orientations if the measured polarizations are significantly contaminated by interstellar polarization. Even should there be contaminating interstellar polarization, however, our observations securely show that disks in young binaries are not perfectly coplanar.

Theories of the time evolution of disk inclinations predict that large initial tilts should decay on a timescale shorter than the age of the systems observed here but that any remaining tilt takes much longer to decay. Thus, it is possible that binaries form with large relative disk inclinations but that those tilts have already decayed to the small but nonzero relative disk inclinations consistent with our observations. If this explanation of our findings is correct, binaries with separations of several thousand AU (wider than those observed here) should still exhibit their primordial relative inclinations.

If the similar disk inclinations of the binaries are due to evolution of initially larger disk inclinations, it is plausible that the misaligned disks in triple systems are the result of the much greater timescale necessary for aligning the orbital angular momentum of the close pair with that of the wide companion; such alignment is necessary for all three circumstellar disks to be aligned with each other.

If the small relative disk inclinations observed here also correspond to small tilts relative to the binary orbital plane, then planetary systems that may form in these binaries should be stable on $10^{9}$ yr timescales (Pendleton \& Black 1983; Innanen et al. 1997; Holman \& Wiegert 1999), suggesting that wide binaries are a hospitable environment for the formation and evolution of planetary systems and that these systems should survive as long as it took life to arise on Earth.

We thank Matthew Bate, Chris Burns, David Cohen, Phil Everson, Matt Holman, and Steve Lubow for useful and stimulating discussions. We also thank the staff of UKIRT, in particular Antonio Chrysostomou, for excellent support before, during, and after our observations. We thank Alyssa Goodman for supplying interstellar polarization data in electronic form. We thank an anonymous referee whose comments improved the presentation of our paper. This research has made use of NASA's Astrophysics Data System, and of the SIMBAD database, operated at CDS, Strasbourg, France. We gratefully acknowledge support of this work by grants AST 99-96278 and AST 03-07830 from the National Science Foundation and by a James Michener fellowship from Swarthmore College.
Arce, H. G., Goodman, A. A., Bastien, P., Manset, N., \& Sumner, M. 1998, ApJ, 499, L93

Artymowicz, P., \& Lubow, S. H. 1994, ApJ, 421, 651

Bastien, P. 1985, ApJS, 59, 277

Bate, M. R., Bonnell, I. A., Clarke, C. J., Lubow, S. H., Ogilvie, G. I., Pringle, J. E. \& Tout, C. A. 2000, MNRAS, 317, 773

Bertout, C., Robichon, N., \& Arenou, F. 1999, A\&A, 352, 574

Bonnell, I., \& Bastien, P. 1993, ApJ, 406, 614

Desch, S. J., \& Mouschovias, T. C. 2001, ApJ, 550, 314

Duchêne, G., \& Ménard, F. 2004, in Open Issues in Local Star Formation, ed. J. Lépine \& J. C. Gregório-Hetem (Dordrecht: Kluwer), 287

Duchêne, G., Monin, J.-L., Bouvier, J., \& Ménard, F. 1999, A\&A, 351, 954

Dutrey, A. 2001, in IAU Symp. 200, Birth and Evolution of Binary Stars, ed. B. Reipurth \& H. Zinnecker (San Francisco: ASP), 219

Dutrey, A., Guilloteau, S., Duvert, G., Prato, L., Simon, M., Schuster, K., \& Ménard, F. 1996, A\&A, 309, 493

Dyck, H. M., Forrest, W. J., Gillett, F. C., Stein, W. A., Gehrz, R. D., Woolf, N. J., \& Shawl, S. J. 1971, ApJ, 165, 57

Fekel, F. C. 1981, ApJ, 246, 879

\section{REFERENCES}

Galli, D., \& Shu, F. H. 1993, ApJ, 417, 220

Gehrels, T. 1974, in IAU Colloq. 23, Planets, Stars, and Nebulae Studied with Photopolarimetry, ed. T. Gehrels (Tucson: Univ. Arizona Press), 168 Gerakines, P. A., Whittet, D. C. B., \& Lazarian, A. 1995, ApJ, 455, L171 Ghez, A. M., Neugebauer, G., \& Matthews, K. 1993, AJ, 106, 2005 Goodman, A. A., Bastien, P., Ménard, F., \& Myers, P. C. 1990, ApJ, 359, 363 Hall, S. M., Clarke, C. J., \& Pringle, J. E. 1996, MNRAS, 278, 303 Heiles, C., Goodman, A. A., McKee, C. F., \& Zweibel, E. G. 1993, in Protostars and Planets III, ed. E. Levy \& J. I. Lunine (Tucson: Univ. Arizona Press), 279

Herbig, G. H., \& Bell, K. R. 1988, Lick Obs. Bull. 1111

Holman, M. J., \& Wiegert, P. A. 1999, AJ, 117, 621

Innanen, K. A., Zheng, J. Q., Mikkola, S., \& Valtonen, M. J. 1997, AJ, 113,1915

Jensen, E. L. N., \& Akeson, R. L. 2003, ApJ, 584, 875

Jensen, E. L. N., Koerner, D. W., \& Mathieu, R. D. 1996, AJ, 111, 2431 Jensen, E. L. N., Mathieu, R. D., \& Fuller, G. A. 1994, ApJ, 429, L29 1996, ApJ, 458, 312

Jones, T. J. 1989, ApJ, 346, 728

Koerner, D. W., \& Sargent, A. I. 1995, AJ, 109, 2138 
Köhler, R., \& Brandner, W. 2001, in IAU Symp. 200, Birth and Evolution of Binary Stars, ed. B. Reipurth \& H. Zinnecker (San Francisco: ASP) 147

Koresko, C. D. 1998, ApJ, 507, L145

Larwood, J. D., Nelson, R. P., Papaloizou, J. C. B., \& Terquem, C. 1996, MNRAS, 282, 597

Lee, C. W., \& Myers, P. C. 1999, ApJS, 123, 233

Leinert, C., Zinnecker, H., Weitzel, N., Christou, J., Ridgway, S. T., Jameson, R., Haas, M., \& Lenzen, R. 1993, A\&A, 278, 129

Lubow, S. H., \& Ogilvie, G. I. 2000, ApJ, 538, 326

Martin, P. G., et al. 1992, ApJ, 392, 691

Mathieu, R. D., Ghez, A. M., Jensen, E. L. N., \& Simon, M. 2000, in Protostars and Planets IV, ed. V. Mannings, A. P. Boss, \& S. S. Russell (Tucson: Univ. Arizona Press), 703

McCaughrean, M. J., Stapelfeldt, K. R., \& Close, L. M. 2000, in Protostars and Planets IV, ed. V. Mannings, A. P. Boss, \& S. S. Russell (Tucson: Univ. Arizona Press), 485

McKee, C. F., Zweibel, E. G., Goodman, A. A., \& Heiles, C. 1993, Protostars and Planets III, ed. E. Levy \& J. I. Lunine (Tucson: Univ. Arizona Press), 327

Moneti, A., \& Zinnecker, H. 1991, A\&A, 242, 428

Monin, J.-L., Ménard, F., \& Duchêne, G. 1998, A\&A, 339, 113

Monin, J., Ménard, F., \& Peretto, N. 2002, in The Origins of Stars and Planets, ed. J. F. Alves \& M. J. McCaughrean (Berlin: Springer), 121

Myers, P. C., Fuller, G. A., Goodman, A. A., \& Benson, P. J. 1991, ApJ, 376,561

Naghizadeh-Khouei, J., \& Clarke, D. 1993, A\&A, 274, 968

Osterloh, M., \& Beckwith, S. V. W. 1995, ApJ, 439, 288

Papaloizou, J. C. B., \& Terquem, C. 1995, MNRAS, 274, 987

Pendleton, Y. J., \& Black, D. C. 1983, AJ, 88, 1415

Prato, L., \& Simon, M. 1997, ApJ, 474, 455
Reipurth, B., \& Zinnecker, H. 1993, A\&A, 278, 81

Simmons, J. F. L., \& Stewart, B. G. 1985, A\&A, 142, 100

Simon, M., Chen, W. P., Howell, R. R., Benson, J. A., \& Slowik, D. 1992, ApJ, 384, 212

Simon, M., et al. 1995, ApJ, 443, 625

Stapelfeldt, K. R., Krist, J. E., Ménard, F., Bouvier, J., Padgett, D., \& Burrows, C. 1998, ApJ, 502, L65

Stephens, M. A., 1974, J. Am. Stat. Assoc., 69, 730

Sterzik, M. F., \& Tokovinin, A. A. 2002, A\&A, 384, 1030

Stewart, B. G. 1991, A\&A, 246, 280

Tachihara, K., Mizuno, A., \& Fukui, Y. 2000, ApJ, 528, 817

Tamura, M., \& Sato, S. 1989, AJ, 98, 1368

Tokovinin, A. A. 1993, Astron. Lett., 19, 383

van Albada, T. S. 1968, Bull. Astron. Inst. Netherlands, 20, 73

Vrba, F. J., Coyne, G. V., \& Tapia, S. 1993, AJ, 105, 1010

Vrba, F. J., Strom, S., \& Strom, K. 1976, AJ, 81, 958

Wardle, J. F. C., \& Kronberg, P. P. 1974, ApJ, 194, 249

Welty, A. D. 1995, AJ, 110, 776

White, R. J., \& Ghez, A. M. 2001, ApJ, 556, 265

Whitney, B. A., \& Hartmann, L. 1992, ApJ, 395, 529 . 1993, ApJ, 402, 605

Whittet, D. C. B., Gerakines, P. A., Hough, J. H., \& Shenoy, S. S. 2001, ApJ, 547, 872

Whittet, D. C. B., Martin, P. G., Hough, J. H., Rouse, M. F., Bailey, J. A., \& Axon, D. J. 1992, ApJ, 386, 562

Wolf, S., Stecklum, B., \& Henning, T. 2001, in IAU Symp. 200, Birth and Evolution of Binary Stars, ed. B. Reipurth \& H. Zinnecker (San Francisco: ASP), 295

Wyatt, S. P., \& Brown, F. G. 1955, AJ, 60, 415

Yudin, R. V. 2000, A\&AS, 144, 285 